\title{
Human Medial Temporal Lobe Damage Can Disrupt the Perception of Single Objects
}

\author{
Andy C. H. Lee and Sarah R. Rudebeck \\ Department of Experimental Psychology, University of Oxford, Oxford OX1 3UD, United Kingdom
}

The idea that the medial temporal lobe (MTL), traditionally viewed as an exclusive memory system, may also subserve higher-order perception has been debated fiercely. To support this suggestion, monkey and human lesion studies have demonstrated that perirhinal cortex damage impairs complex object discrimination. The interpretation of these findings has, however, been disputed because these impairments may reflect a primary deficit in MTL-mediated working memory processes or, in the case of human patients, undetected damage to visual processing regions beyond the MTL. To address these issues, this study investigated object perception in two human amnesic patients who were chosen on the basis of their lesion locations and suitability for detailed neuroimaging investigation. A neuropsychological task with minimal working memory demands was administered in which participants assessed the structural coherency of single novel objects. Critically, only the patient with perirhinal atrophy was impaired. Moreover, volumetric and functional neuroimaging data demonstrated that this deficit cannot be attributed to the dysfunction of visual cortical areas. Additional analyses of eye-movement patterns during the perceptual task revealed an inability of this patient to detect structural incoherency consistently. This study uses a combination of techniques to provide strong evidence that the perirhinal cortex subserves perception and suggests that the MTL perceptual-mnemonic debate cannot be dismissed on the basis of anatomy or a working memory impairment.

\section{Introduction}

Traditional theory states that the medial temporal lobe (MTL), including the hippocampus (HC), entorhinal cortex (ERC), perirhinal cortex (PRC), and parahippocampal cortex (PHC), functions as a dedicated long-term declarative memory system (Squire et al., 2004). Recent work suggests, however, that the MTL may also be critical for higher-order perception (Buckley and Gaffan, 2006; Murray et al., 2007).

At the center of this controversy are convergent studies that have demonstrated visual discrimination deficits in nonhuman animals and humans with MTL damage, with HC lesions impairing complex spatial discrimination, and PRC damage producing complex object discrimination deficits (Buckley et al., 2001; Bussey et al., 2002, 2003; Barense et al., 2005, 2007; Lee et al., 2005a,b, 2006, 2007; Bartko et al., 2007a,b). The interpretation and implications of these findings have, however, been debated for several reasons. First, these discrimination impairments have not been replicated by proponents of a dedicated MTL memory system (Buffalo et al., 1998, 1999; Stark and Squire, 2000; Levy et al., 2005; Shrager et al., 2006). Second, studies that have reported

\footnotetext{
Received Jan. 8, 2010; revised March 2, 2010; accepted March 27, 2010.

This work was supported by Wellcome Trust Grant 082315 (A.C.H.L.), an Oriel College Hayward Junior Research Fellowship (A.C.H.L.), and the Alzheimer's Research Trust (United Kingdom). We thank all the participants for their time, Kathryn Atherton for help with data collection, Prof. David Gaffan, Dr. Mark Baxter, Dr. Mark Buckley, Dr. Rhys Davies, and Prof. Kim Graham for helpful discussion, and Steven Knight (University of Oxford Centre for Clinical Magnetic Resonance Research, 0xford, UK) and staff at the Magnetic Resonance Imaging Services, Addenbrooke's Hospital (Cambridge, UK) for MRI assistance. Task stimulus images courtesy of Michael J. Tarr, Carnegie Mellon University, www.tarrlab.org/.

Correspondence should be addressed to Dr. Andy Lee, Department of Experimental Psychology, University of Oxford, South Parks Road, Oxford OX1 3UD, UK. E-mail: andy.lee@psy.ox.ac.uk.

DOI:10.1523/JNEUROSCI.0116-10.2010

Copyright $\odot 2010$ the authors $\quad 0270-6474 / 10 / 306588-07 \$ 15.00 / 0$
}

visual discrimination deficits after MTL damage have all relied on neuropsychological tasks that require participants to compare multiple simultaneously presented images, such as oddity judgment. Thus, it is plausible that memory and perception are confounded in these tests, and poor performance may reflect a deficit in MTL-mediated working memory processes (Ranganath and D'Esposito, 2001; Olson et al., 2006; Hartley et al., 2007) or the failure to use long-term memory representations (Shrager et al., 2006) rather than a perceptual problem. Finally, volumetric magnetic resonance imaging (MRI) analyses were previously not possible in those amnesic patients that demonstrated visual discrimination problems. The MTL perceptual-mnemonic debate has, therefore, been dismissed on the basis that the original lesion descriptions provided for these patients were inadequate and thus failed to detect potential damage to structures beyond the MTL (Shrager et al., 2006), for instance, lateral temporal cortex or extrastriate visual areas.

We aimed to resolve these issues by investigating whether perirhinal damage impairs the processing of objects presented in isolation. To counter anatomical concerns, we focused on two amnesic patients who, from previous visual ratings (Lee et al., 2005a), do not appear to have lateral temporal cortex damage and, more importantly, are suitable for additional MRI investigations, thus allowing volumetric lesion analyses and functional MRI of extrastriate visual areas. By adapting a classic object decision paradigm (Schacter et al., 1990), the patients and matched healthy participants were asked to assess the structural coherency of single drawings of novel objects. Performance on this task was compared with that on a test in which participants made same/ different judgments of two novel objects presented simultaneously (a task that demanded the comparison of multiple 
Table 1. Individual patient's Z scores for each measured brain region in the left and right hemispheres

\begin{tabular}{|c|c|c|c|c|c|c|c|c|c|c|}
\hline & $\begin{array}{l}\text { Temporopolar } \\
\text { cortex }\end{array}$ & Amygdala & Entorhinal cortex & Perirhinal cortex & Hippocampus & $\begin{array}{l}\text { Parahippocampal } \\
\text { cortex }\end{array}$ & $\begin{array}{l}\text { Anterior fusiform } \\
\text { gyrus }\end{array}$ & $\begin{array}{l}\text { Posterior fusiform } \\
\text { gyrus }\end{array}$ & $\begin{array}{l}\text { Anterior lateral } \\
\text { temporal cortex }\end{array}$ & $\begin{array}{l}\text { Posterior lateral } \\
\text { temporal cortex }\end{array}$ \\
\hline \multicolumn{11}{|l|}{ Left } \\
\hline $\mathrm{HC} 3$ & 1.06 & 1.86 & 1.44 & 0.18 & -4.78 & -0.74 & -0.57 & 0.39 & -0.43 & 0.49 \\
\hline MTL3 & -0.17 & -3.23 & -4.72 & -2.19 & -3.46 & -3.59 & -1.08 & -1.36 & -0.58 & -0.45 \\
\hline \multicolumn{11}{|l|}{ Right } \\
\hline $\mathrm{HC} 3$ & 0.43 & 0.94 & 0.31 & -0.90 & -3.92 & -0.73 & -0.09 & 0.78 & -0.33 & -0.53 \\
\hline MTL3 & -7.01 & -9.94 & -4.63 & -3.21 & -6.66 & -2.84 & -3.31 & -1.87 & -5.27 & -1.41 \\
\hline
\end{tabular}

Bold indicates a significantly reduced volume compared with the healthy control group $(Z<-1.96)$.

stimuli but not higher-order object perception). Crucially, eye movements were recorded to gain detailed insight into the patients' performance.

\section{Materials and Methods}

Participants. Given the importance of well defined lesions and the potential influence of dysfunction in structurally intact visual areas, we only selected amnesic patients with circumscribed MTL damage who were suitable for extensive MRI investigations. Accordingly, two patients were studied, one previously identified using qualitative visual ratings as having predominant bilateral $\mathrm{HC}$ damage (patient $\mathrm{HC} 3$, female, 50 years old, education of 10 years), and the other with a larger bilateral MTL lesion, including the HC and PRC (patient MTL3, female, 64 years old, education of 10 years) (Lee et al., 2005a). Patient HC3 suffered brain damage after carbon monoxide-induced hypoxia, whereas patient MTL suffered from herpes encephalitis. Patient HC3 demonstrates intact semantic memory (category comprehension, 64 of 64; pyramids and palm trees, 52 of 52) but poor episodic memory recall (logical memory immediate recall, 22 of 75; delayed recall, 4 of 50; Rey-Osterrieth Complex Figure delayed recall, 3 of 36) and impaired recognition memory for prose (logical memory recognition, 19 of 30) but not scenes or faces (Warrington Recognition Memory Test scenes, 26 of 30; faces, 44 of 50). Patient MTL3 possesses severe episodic memory recall (logical memory immediate recall, 13 of 75; delayed recall, 4 of 50; Rey-Osterrieth Complex Figure delayed recall, 4.5 of 36) and recognition deficits (logical memory recognition, 23 of 30; Warrington Recognition Memory Test scenes, 13 of 30; faces, 30 of 50), as well as a mild semantic memory impairment (category comprehension, 54 of 64; pyramids and palm trees, 46 of 52). Both patients perform within the normal range on the Rey-Osterrieth Complex Figure copy condition, as well as all subtests of the Visual Object and Space Perception Battery.

Critically, although patients HC3 and MTL3 perform within the normal range on standard visuoperceptual tests, they exhibit different profiles of performance on experimental oddity judgment tasks (supplemental Fig. 1 , available at www.jneurosci.org as supplemental material) in which the odd-one-out must be selected from an array of simultaneously presented images. Both patients demonstrate normal accuracy on difficult oddity judgment tasks involving simple visual stimuli (color oddity: patient HC3, 70.77\%; patient MTL3, 83.08\%; size oddity: patient HC3, 82.86\%; patient MTL3, 82.86\%; all $t_{(9)}<1 ; p>0.4$ compared with matched controls using two-tailed modified $t$ test for small samples) (Crawford and Howell, 1998), but both are poor at complex spatial scene oddity (patient HC3, 45.16\%; patient MTL3, 51.61\%; both $t<-3$; $p<0.05$ ), underlining the importance of the hippocampus in complex spatial processing. In support of a role for the perirhinal cortex in complex object perception, patient MTL3 is also impaired at complex object oddity judgment (familiar objects: patient HC3, 82.86\%, $t_{(9)}=-0.087, p=0.93$; patient MTL3, 51.43\%, $t_{(9)}=-2.54, p=0.032$; novel objects: patient HC3, 74.29\%, $t_{(9)}=-1.18, p=0.27$; patient MTL3, 17.14\%, $t_{(9)}=$ $-5.75, p \leq 0.001$ ) (data from Lee et al., 2005b; Barense et al., 2007).

For behavioral assessment, two groups of six neurologically healthy female participants were recruited to compare with patient HC3 (mean age, 51.85 years; education, 10.83 years) and patient MTL3 (mean age, 65.23 years; education, 11.33 years). There was no significant difference, however, between these two groups in terms of accuracy or response times on the possible/impossible judgment task (accuracy, $t_{(10)}=0.29$, $p=0.78$; response time, $\left.t_{(10)}=0.35, p=0.73\right)$ or the same/different judgment test (accuracy, $t_{(10)}=1.12, p=0.29$; response time, $t_{(10)}=$ $1.48, p=0.17)$. Subsequently, all control participants were combined into a single larger cohort (mean \pm SD age, $58.54 \pm 8.23$ years; mean \pm SD education, $11.08 \pm 1.00$ years) for comparison with both amnesic cases. There was no significant difference between each patient and this larger control group in terms of age or years of education (all $t_{(11)}<1$, $p>0.3)$.

All participants gave informed written consent. This research received ethical approval from the Oxfordshire Research Ethics Committee (07/ H0604/115; 08/H0606/133), the University of Oxford Central University Research Ethics Committee (MSD/IDREC/C1/2008/65), and the Cambridgeshire Health Authority Local Research Ethics Committee.

Volumetric assessment of patient lesions. T1 structural MRI scans were acquired for each patient (patient HC3 at age 46 years, patient MTL at 59 years) and 11 female controls (mean \pm SD age, $55.27 \pm 10.80$ years; no significant difference between controls and patients, both $t_{(10)}<1, p>$ 0.4) using a 1.5T GE Signa scanner at the MRI Department, Addenbrooke's Hospital (Cambridge, UK). The matched healthy controls for the volumetric analyses did not participate in the behavioral assessment or functional MRI parts of this study.

Consistent with other recent studies (Tsivilis et al., 2008), volumetric analyses were conducted by a single observer (A.C.H.L.). Regions of interest (ROIs) were manually traced on coronal slices in each hemisphere using MRIcron software (Rorden and Brett, 2000). All scans were $256 \times 122 \times 256$ in size, with voxel dimensions $0.86 \times 1.80 \times 0.86 \mathrm{~mm}$. Temporopolar cortex (TPC), ERC, and PRC were defined using the Insausti protocol (Insausti et al., 1998), whereas the hippocampus (HC) and amygdala were identified with the Mayo Clinic method (Watson et al., 1997). The fusiform gyrus was measured from the slice coinciding with the anterior boundary of the PRC, whereas the PHC was measured from the slice immediately after the posterior boundary of the PRC. The posterior boundaries of both these regions were delineated on the slice coinciding with the posterior extent of the HC. A measure for lateral temporal cortex was derived by obtaining a measure for the gray matter of the entire temporal cortex from the tip of the TPC to the posterior end of the HC and subtracting the volumes for TPC, ERC, PRC, PHC, and the fusiform gyrus. The fusiform gyrus and lateral temporal volumes were further subdivided into two by measuring separately the slices anterior and posterior to the midpoint, the reason being that the anterior portion of the temporal lobe toward the TPC is not known to be associated with visual processing (i.e., as part of the ventral visual processing stream). All measured volumes were corrected for intracranial volume, which was determined by drawing around the brain tissue in all coronal slices, including gray and white matter and ventricular space and excluding the brainstem below the level of the pons. Repeatability was assessed by re-measuring all ROIs in eight of the cases at least 6 weeks after the first measurement (both patients and 6 of 11 controls) and calculating intraclass correlation coefficients. These revealed good repeatability in all areas (all $r>0.9$ ) (supplemental Table 1, available at www.jneurosci.org as supplemental material).

Calculated $Z$ scores for every measured brain region (supplemental Fig. 2, available at www.jneurosci.org as supplemental material) for each patient compared with the healthy controls revealed that patient $\mathrm{HC} 3$ has significant bilateral HC damage $(Z$ score $<-1.96)$, with no significant damage beyond this structure (Table 1). Patient MTL3 has significant 


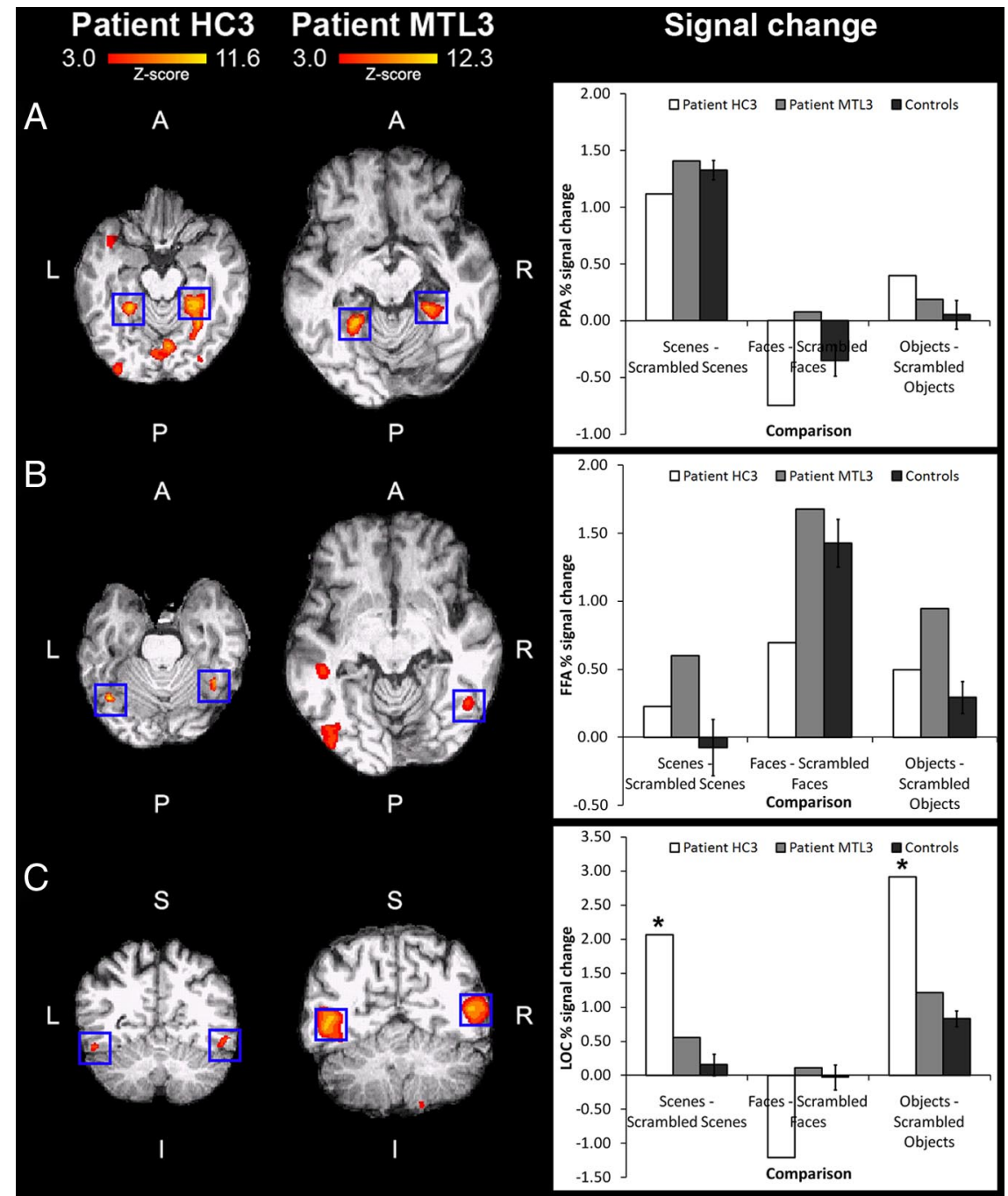

Figure 1. The PPA (A), FFA (B), and LOC $(\boldsymbol{C})$ in each patient (blue squares) as identified by the following contrasts: scenes (faces + objects); faces - (scenes + objects); and objects - (scenes + faces). Activity thresholded at $p<0.001$ uncorrected and rendered on each patient's structural scan. The patterns of activity ( \pm SE) at the most significant voxel in these regions are shown. $A$, Anterior; $P$, posterior; $L$, left; $R$, right; $S$, superior; I, inferior. ${ }^{*}$ indicates significantly different from controls, $p<0.01$.

damage in a number of regions within and beyond the MTL. Importantly, although there is significant damage to the PRC and HC bilaterally, there is no significant damage to the fusiform gyrus or lateral temporal cortex in the left hemisphere (anteriorly or posteriorly) or to the right posterior fusiform gyrus or right posterior lateral temporal cortex, suggesting intact posterior visual regions.

Functional neuroimaging of extrastriate visual areas. The integrity of the parahippocampal place area (PPA) (Epstein and Kanwisher, 1998), fusiform face area (FFA) (Kanwisher et al., 1997), and lateral occipital complex (LOC) (Malach et al., 1995) was assessed, each suggested to be important for perceiving spatial scenes, faces, and objects, respectively. One four-dimensional MRI dataset was acquired for each patient (patient $\mathrm{HC} 3$ at age 50 years; patient MTL3 at age 64 years) and nine controls (five female; mean \pm SD age, $57.38 \pm 10.41$ SD; no significant difference between controls and patients, both $\left.t_{(8)}<1, p>0.5\right)$ on a 3 T Sonata TRIO (Siemens) with a 12-channel head coil at the University of Oxford Centre for Clinical Magnetic Resonance Research using an echo planar imaging pulse (EPI) sequence to acquire $\mathrm{T} 2^{*}$-weighted volumes with blood oxygen level-dependent contrast. The matched healthy controls for this part of the study did not participate in the volumetric analyses or behavioral assessment. Axial-oblique slices angled away from the eye balls were obtained to prevent image ghosting [voxel resolution, $3 \times 3 \times 3$ $\mathrm{mm}$; matrix size, $64 \times 64 \times 46$; repetition time (TR), 3 s; echo time $(\mathrm{TE}), 30$ ms; field of view (FOV), $192 \times 192 \mathrm{~mm}$; flip angle, $89^{\circ}$. The EPI scan was $512 \mathrm{~s}$ in duration, consisting of 180 scans and four dummy scans at the start to allow the MR signal to reach an equilibrium state. A T1 structural scan and magnetic field maps were also obtained for each individual, the former with a three-dimensional magnetization-prepared rapid-acquisition gradient echo sequence (voxel resolution, $1 \times$ $1 \times 1 \mathrm{~mm}$; TR, $2.04 \mathrm{~s}$; TE, $4.7 \mathrm{~ms}$; flip angle, $8^{\circ}$; FOV, $192 \times 192$ mm; matrix size, $174 \times 192 \times$ 192 ) and the latter were with a dual-echo twodimensional gradient echo sequence with echoes at 5.19 and $7.65 \mathrm{~ms}$ (voxel resolution, $3.5 \times 3.5 \times$ $3.5 \mathrm{~mm}$; TR, $0.49 \mathrm{~s}$; flip angle, $60^{\circ}$; FOV, $224 \times$ $224 \mathrm{~mm}$; matrix size, $64 \times 64 \times 44$ ).

During scanning, a functional localizer task (Epstein and Kanwisher, 1998) was administered to identify extrastriate visual areas. Fifteen-second blocks of grayscale images of faces, scenes, objects, scrambled faces, scrambled scenes, and scrambled objects were presented. There were 20 images in each block, with each image being presented for $300 \mathrm{~ms}$ (interstimulus interval, $450 \mathrm{~ms}$ ). There were four blocks of each stimulus type, and the order of the different blocks was pseudorandomized. The participants were instructed to press a specified button on a response box held in the right hand when they noticed a repeating image (i.e., one-back task). A fixation cross was presented every four blocks throughout the task $(9 \times 15 \mathrm{~s})$, as well as a brief task instruction screen $(8 \times 3$ s) designed to remind the patients of what they were required to do (supplemental Fig. 3, available at www.jneurosci.org as supplemental material).

The stimuli were presented with a computer program written using the Presentation software package (Neurobehavioral Systems). This program was run on a desktop computer connected to a liquid crystal display (LCD) projector $(1024 \times 768$ pixel resolution $)$ that projected onto a white screen situated at the head of the MRI subject bed. The screen could be seen via an angled mirror placed above the subject's eyes in the scanner.

Imaging data preprocessing and analyses were performed with the FMRI Expert Analysis Tool (FEAT version 5.98) part of the FMRIB (Oxford University Centre for Functional MRI of the Brain) Software Library (www.fmrib.ox.ac.uk/fsl) (Smith et al., 2004). Each individual's data were first preprocessed by the following: (1) realigning all images to the central volume using rigid body registration (Jenkinson et al., 2002); (2) unwarping the EPI data using field-map data to correct for distortions attributable to magnetic field inhomogeneities (Jenkinson et al., 2002); (3) segmenting brain matter from non-brain matter using a mesh deformation approach; (4) applying spatial smoothing using a Gaussian kernel (fullwidth half-maximum, $6.0 \mathrm{~mm}$ ); (5) implementing grand-mean intensity normalization of the dataset by a single multiplicative factor; (6) performing high-pass temporal filtering using Gaussian-weighted least-squares straight line fitting with $\sigma=150 \mathrm{~s}$; (7) using independent component analysis-based exploratory data analysis to identify and remove unexpected artifacts (Beckmann and Smith, 2004); and finally (8) registering each patient's EPI dataset to their T1 structural scan using affine registration (Jenkinson and Smith, 2001; Jenkinson et al., 2002).

For statistical analyses, a general linear model was fit in prewhitened data space with seven explanatory variables (corresponding to the onsets and durations of the seven stimulus conditions) and their temporal derivatives (Woolrich et al., 2001). Each explanatory variable was convolved with a 
double-gamma function to model the human hemodynamic response function and temporally filtered with the same high-pass filter as the data. Thus, a parameter estimate image was created for each explanatory variable as well as a number of planned contrasts to examine differential activity across experimental conditions, including the following: (1) the main effect of viewing intact scenes to locate the PPA: scenes - (faces + objects); (2) the main effect of viewing intact faces to locate the FFA: faces - (scenes + objects); and (3) the main effect of viewing intact objects to locate the LOC: objects (scenes + faces). Because single-subject data were analyzed and there were clear predictions regarding regions of activity associated with the viewing of scenes, faces, and objects, significant regions of activity were identified with a threshold of $p<0.001$ uncorrected for multiple comparisons. To illustrate the pattern of activity in each extrastriate visual region, the mean percentage signal change was extracted from the most significant voxel for all seven conditions, thus allowing the comparison between each intact stimulus category and its scrambled counterpart (i.e., scenes vs scrambled scenes, faces vs scrambled faces, objects vs scrambled objects).

Functionally defined extrastriate visual areas were identified in both patients in at least one hemisphere (Fig. 1), including the PPA bilaterally in both patients (patient HC3, left: 55 voxels; maximum $Z$ score of 7.41; right: 276 voxels, maximum $Z$ score of 8.43; patient MTL3, left: 41 voxels, maximum $Z$ score of 5.36; right: 28 voxels; maximum $Z$ score of 6.35 ), the FFA in both hemispheres in patient HC3 (left: 18 voxels; maximum $Z$ score of 5.83; right: 33 voxels; maximum $Z$ score of 6.40 ), in the right hemisphere in patient MTL3 (15 voxels; maximum $Z$ score of 5.25), and finally the LOC bilaterally in both patients (patient HC3, left: 22 voxels; maximum $Z$ score of 4.21; right: 61 voxels; maximum $Z$ score of 4.59; patient MTL3, left: 274 voxels; maximum $Z$ score of 7.95; right: 222 voxels; maximum $Z$ score of 7.53$)$.

The PPA ( $Z$ score range of 4.21-8.99), FFA ( $Z$ score range of $5.20-$ 8.87 ), and LOC ( $Z$ score range of 4.42-6.61) were also successfully located in most healthy controls, the exceptions being the LOC in two cases and the FFA in a third participant. The failure to find these regions is not entirely surprising because previous work has also failed to find functionally defined extrastriate regions in one or both hemispheres in a proportion of neurologically healthy participants (Malach et al., 1995; Kanwisher et al., 1997). Extracting the percentage signal change in these regions at the most significant voxel (intact vs scrambled stimuli) confirmed that the pattern of activity across the different stimulus categories in all of these areas in the patients was highly similar to that of controls (Fig. 1). Two-tailed modified $t$ tests for small samples (Crawford and Howell, 1998) revealed no significant differences in the levels of activity between either patient and controls in the PPA (both patients all $t_{(8)} \leq 1.1, p>0.3$ ) or FFA (both patients all $t_{(7)} \leq 1.9$, $p>0.1$ ) or between patient MTL3 and the controls in the LOC (all $t_{(6)} \leq 1.2$, $p>0.2)$. However, although there was no significant difference between patient HC3 and controls in the LOC for the signal change associated with the viewing of intact versus scrambled faces $\left(t_{(6)}=-2.24, p=\right.$ $0.066)$, the change in activity was significantly greater in patient HC3 than that in controls for intact versus scrambled objects $\left(t_{(6)}=6.31, p \leq\right.$ $0.001)$ and intact versus scrambled scenes $\left(t_{(6)}=4.19, p=0.0057\right)$.

Behavioral assessment. Testing was conducted on a Tobii T120 system (Tobii Technology), which consists of a 17 inch LCD monitor $(1280 \times$ 1024 pixel resolution) with an inbuilt $120 \mathrm{~Hz}$ infrared eye tracker, connected to a laptop computer. Stimulus presentation, response recording, and eye-movement tracking were managed by the Tobii Studio version 1.5 software package.

Two tasks were administered: (1) possible/impossible object judgment and (2) same/different object judgment (Fig. $2 A$ ). In the former, subjects were presented with 40 successive black and white line drawings of novel objects, half of which contained a single region that was structurally incoherent and thus "impossible" in the real world ("Impossible1" and "Possible" figures from Williams and Tarr, 1997). Participants were instructed to determine whether each object was "possible" or "impossible" and to make their response by a prespecified key press. In the same/ different judgment task, 48 successive pairs of black and white line drawings were presented. Each pair consisted of either identical or different objects (24 trials each; comprising "Impossible1," "Impossible3," and "Possible" figures from Williams and Tarr, 1997). Participants indicated whether each pair of objects was identical or different by pressing a
A (i)

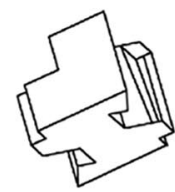

(ii)

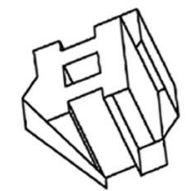

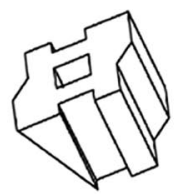
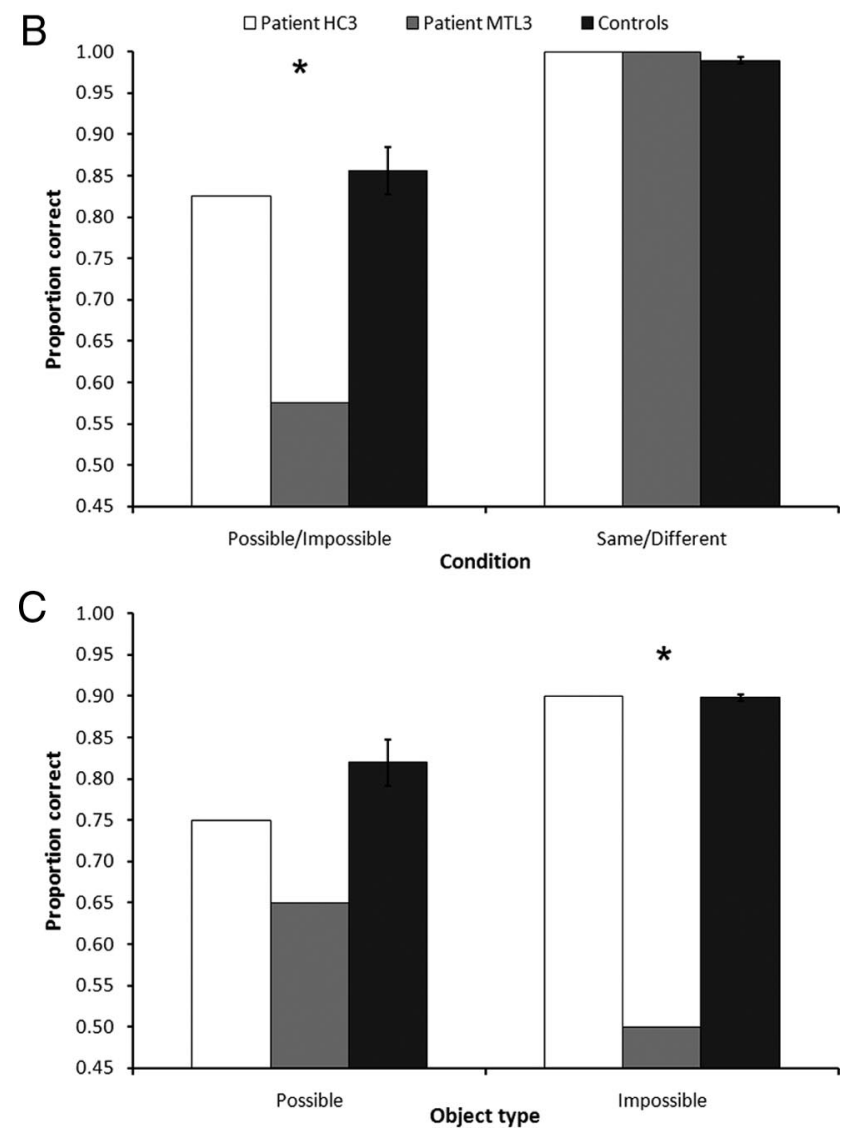

Figure 2. Sample trial from the possible/impossible (Ai) and same/different (Aii) judgment tasks. $\boldsymbol{B}$, Overall proportion correct for the possible/impossible and same/different tasks. C, Proportion correct for possible and impossible objects ( \pm SE). * indicates significant impairment, $p<0.01$.

prespecified key. In both tasks, a $2 \mathrm{~s}$ fixation cross was presented between trials and no object was repeated (each trial was unique). Moreover, no stimulus appeared in both tests, thus reducing the influence of long-term memory processes. All object stimuli were presented until a response was made, and participants were encouraged to respond as quickly as possible without compromising accuracy. Detailed instructions and separate practice blocks (with different stimuli) were given before actual test administration.

Eye-movement data were filtered and analyzed using algorithms implemented in Tobii Studio. Full details of this approach are available elsewhere (www.tobii.com) but are summarized here: (1) all missing gaze point data (e.g., attributable to eye blinks) are filled in using interpolation of acquired samples; (2) for each gaze point in the gaze data sequence, a position difference vector is calculated using the means of the sample points within two adjacent sliding time windows of $42 \mathrm{~ms}$ duration; (3) a position difference vector $>35$ pixels (indicating an abrupt change in gaze position) is classified as a saccade, whereas vectors below this threshold are treated as points of fixation; and (4) the spatial position of the fixations between saccades is estimated by calculating the median of all samples in the interval.

For the possible/impossible judgment task, eye movements were analyzed for those trials in which an impossible object was presented because a significant patient deficit was found for these items (see Results). The structurally incoherent region of each impossible object was delineated as an ROI, and the fixation durations within and outside of this area 
relative to the total fixation time for the entire object were calculated. A difference score was obtained by subtracting the proportion of fixation time outside of the structurally incoherent region from the proportion of fixation time within this area. For the same/ different judgment task, eye movements were analyzed separately for correct trials for which identical and different objects were presented. In both cases, the left- and right-sided objects were delineated as separate ROIs, and the fixation durations with these areas relative to the total fixation time for the trial were calculated. A difference score was obtained by subtracting the proportion of fixation time on the rightsided object from that on the left-sided object.

The various performance measures for each patient were compared with the control group using two-tailed modified $t$ tests for comparing an individual with a small sample (Crawford and Howell, 1998).

\section{Results}

Patient HC3 had little difficulty on the possible/impossible judgment task (Fig. 2) $\left(t_{(11)}=-0.40, p=0.70\right)$, performing similarly to controls regardless of whether the presented object was possible $\left(t_{(11)}=\right.$ $-0.58, p=0.58)$ or impossible $\left(t_{(11)}=\right.$ 0.022, $p=0.98)$. In contrast, patient MTL3 was significantly impaired on this task $\left(t_{(11)}=-3.61, p=0.0041\right)$, with this deficit being predominantly attributable to a failure to identify impossible objects $\left(t_{(11)}=-4.05, p=0.0012\right)$, with the performance on possible objects not being significantly worse than controls $\left(t_{(11)}=-1.40, p=0.19\right)$. Both patients were able to determine whether two simultaneously presented objects were identical or different (both $t_{(11)}=0.92, p=$ 0.38). Furthermore, the patients' response times did not differ significantly from healthy controls in either task (all $t_{(11)}<1.3$, $p>0.2)$.

Given patient MTL3's difficulties with impossible object trials, her eye movements during these trials were analyzed. This revealed that, for correct response trials (50\% trials), there was no significant difference between patient MTL3 and controls: both spent a greater proportion of time fixating on the region that characterized each item as being impossible compared with the remainder of the image (Fig. 3) $\left(t_{(11)}=-0.97, p=0.35\right)$. In contrast, there was a significant difference between patient MTL3 and controls on incorrect response trials. Whereas controls continued to spend a larger amount of time looking at the structurally incoherent segment of the object, patient MTL3 fixated more on regions beyond this area $\left(t_{(8)}=-2.37, p=0.045\right)$. Despite intact behavioral performance, patient HC3's eye movement patterns were similar to that of patient MTL3: whereas there was no significant difference between patient HC3 and controls on correct trials $(90 \%$ trials) $\left(t_{(11)}=-1.44, p=0.18\right)$, patient HC3 did spend significantly less time looking at the impossible region of each object on incorrect trials $\left(t_{(8)}=-2.44, p=0.041\right)$.

On the same/different task, there was no significant difference between either patient and the control group, with all participants spending approximately the same amount of time looking at the left- and right-sided object on each trial for same (patient HC3, $t_{(11)}=-2.06, p=0.064$; patient MTL3, $t_{(11)}=-0.42, p=0.68$ ) and different (patient HC3, $t_{(11)}=$ $-0.83, p=0.42$; patient MTL3, $\left.t_{(11)}=-0.15, p=0.88\right)$ response trials (Fig. 3 ).

\section{Discussion}

By adapting an object decision paradigm for use in two amnesic patients with detailed lesion analyses, we have demonstrated that MTL damage can impair the perception of single objects. Patient MTL3, who has HC and PRC damage, was profoundly impaired on a task that required participants to process the structural coherency of novel objects presented individually. In contrast, patient HC3, who has HC damage that spares the PRC, performed within the normal range on that task. Neither patient was impaired when making a same/different judgment for two simultaneously presented images.

These findings provide strong evidence that object processing deficits can be elicited in a patient with MTL damage, without the need for a task that demands the comparison of multiple simultaneously presented stimuli. This undermines the possibility that previously described visual discrimination deficits in amnesic patients (which were all in the context of tests that involved multiple stimuli on each trial, e.g., oddity judgment) can be explained entirely by a disruption to MTL-mediated within-trial working memory processes (Ranganath and D'Esposito, 2001; Olson et al., 2006; Hartley et al., 2007). Moreover, the use of trial-unique novel stimuli in the present study also discounts the involvement of longer-term between-trial memory representations (Shrager et al., 2006). Instead, our data underline a critical role for the MTL in higher-order perception (Buckley and Gaffan, 2006; Murray et al., 2007). 
We used volumetric and functional MRI in each patient to rule out the possibility that reported perceptual deficits in these cases may be attributable to damage or dysfunction beyond the MTL. Patient HC3 possesses a selective HC lesion, as well as intact extrastriate visual areas (PPA, FFA, and LOC, as indicated by their presence and profiles of activity). Thus, it is unlikely that patient HC3's difficulties with complex spatial processing (see Materials and Methods) can be explained by damage to regions beyond the MTL, particularly the parahippocampal cortex, which has been implicated in the processing and representation of spatial scenes (Epstein, 2008).

As is common with amnesic patients with larger MTL lesions, patient MTL3 has significant atrophy to multiple regions, including the HC, ERC, PRC, amygdala, and PHC bilaterally, as well as the TPC, anterior fusiform gyrus, and anterior lateral temporal cortex in the right hemisphere (the latter attributable to an essentially absent right anterior temporal lobe). Critically, patient MTL3 does not possess damage to cortical regions that are typically associated with visual processing. There is no significant damage to the posterior fusiform gyrus or posterior lateral temporal cortex in either hemisphere, and functional neuroimaging revealed the presence of an intact PPA, FFA, and LOC, therefore underlining intact basic perception of scene, face, and object stimuli. Additionally, patient MTL3 is unimpaired on difficult size and color discrimination tasks and standard neuropsychological tests of perception (as is patient HC3) (see Materials and Methods), arguing against a general impairment in visual perception. The profile of patient MTL3's brain damage, therefore, converges with (1) monkey studies that have demonstrated complex object discrimination deficits after selective PRC damage (Buckley et al., 2001; Bussey et al., 2002, 2003) and (2) functional neuroimaging studies in healthy human participants that have reported PRC activity during object discrimination tasks (Devlin and Price, 2007; Lee et al., 2008; O’Neil et al., 2009; Barense et al., 2010), to support the idea that the PRC is critical for higher-order object perception.

Patient MTL3 made 50\% errors on impossible object trials and, in contrast to controls, spent a smaller proportion of time viewing the impossible region of each item compared with the remaining structurally coherent areas on these trials. Because patient HC3 demonstrated a similar pattern of eye movements to patient MTL3 and yet exhibited intact performance in identifying impossible objects, it is difficult to conclude whether patient MTL3's eye movements on incorrect impossible object trials are a direct result of abnormal object processing. What is certain, however, is that patient MTL3's deficit in identifying impossible objects cannot be explained by a failure to look at the structurally incoherent region of each impossible item. If anything, it is possible that patient MTL3 (and HC3), having looked at these regions, did not perceive them to be of particular interest and potentially incoherent in structure (indeed, controls reported deliberating over their decision before making a response on incorrect trials). Because patient MTL3 (and HC3) exhibited similar viewing patterns as controls for correct impossible object trials (spending the majority of time looking at the impossible region of each presented item), it appears that, for these trials, patient MTL3 had indeed identified the impossible region of the presented object, reflecting a clear understanding of the task.

Importantly, patient MTL3's deficit in the possible/impossible judgment task cannot be attributed simply to a greater degree of general difficulty of this task compared with the same/different test. There was no significant difference between patient MTL3 and controls in the identification of possible objects, which con- trols found more difficult than the identification of impossible items (in fact, a number of controls performed numerically poorer than patient MTL3 on same/different judgments). Furthermore, patient MTL3 demonstrates intact performance on difficult experimental perceptual tests such as fine size and color discrimination (see Materials and Methods). Therefore, patient MTL3's difficulties on the possible/impossible task likely relate to the significant demand that this test places on object perceptual processing.

The PRC is suggested to play a role in perception by processing complex conjunctions of object features, thus enabling the formation of high-level object representations (Murray and Bussey, 1999). Previous studies have stressed the use of such representations by systematically implementing a large amount of "feature ambiguity" among task stimuli. Feature ambiguity refers to the degree of shared features between simultaneously or successively presented stimuli. The discrimination of featureambiguous objects is thought to rely on the processing of complex object features and has been shown to be sensitive to PRC damage (Bussey et al., 2002, 2003; Barense et al., 2005, 2007; Lee et al., 2005a). Our findings demonstrate that feature ambiguity may not be the only way to place a demand on high-level object representations. The present possible/impossible judgment task requires participants to assess the structural coherency of individual objects by considering how their features combine to form edges and surfaces. We suggest that this necessitates the processing of feature conjunctions and thus the involvement of the PRC, damage to which is the likely cause of patient MTL3's impairment on this task.

By presenting two stimuli on each trial, which could be distinguished using single object features, the same/different judgment task was designed to place a greater demand on working memory than the possible/impossible task, without the need for processing complex object feature conjunctions. The intact performance of both patients on the former, therefore, further emphasizes the fact that visual discrimination deficits after MTL damage cannot be explained entirely by impaired MTL-mediated working memory processes.

Finally, we acknowledge the limitations of the single case approach adopted here. The assessment of only two patients was determined by the need for cases with circumscribed MTL damage who were suitable for additional MRI investigations, the absence of which could cast doubt on the interpretation of any behavioral data. Although the generalizability of our findings may be unclear until proven, it is important to note that the data from both patients meet the predictions of the MTL-perceptual hypothesis (e.g., that only PRC damage should impair complex object processing) and concur with the many studies that have found evidence in favor of this viewpoint. We argue, therefore, that our findings provide additional support for the notion that the MTL does not function as an exclusive memory system but also contributes to higher-order perception, with the PRC being critical for complex object processing.

\section{References}

Barense MD, Bussey TJ, Lee ACH, Rogers TT, Davies RR, Saksida LM, Murray EA, Graham KS (2005) Functional specialization in the human medial temporal lobe. J Neurosci 25:10239-10246.

Barense MD, Gaffan D, Graham KS (2007) The human medial temporal lobe processes online representations of complex objects. Neuropsychologia 45:2963-2974.

Barense MD, Henson RN, Lee ACH, Graham KS (2010) Medial temporal lobe activity during complex discrimination of faces, objects, and scenes: effects of viewpoint. Hippocampus 20:389-401. 
Bartko SJ, Winters BD, Cowell RA, Saksida LM, Bussey TJ (2007a) Perceptual functions of perirhinal cortex in rats: zero-delay object recognition and simultaneous oddity discriminations. J Neurosci 27:2548-2559.

Bartko SJ, Winters BD, Cowell RA, Saksida LM, Bussey TJ (2007b) Perirhinal cortex resolves feature ambiguity in configural object recognition and perceptual oddity tasks. Learn Mem 14:821-832.

Beckmann CF, Smith SM (2004) Probabilistic independent component analysis for functional magnetic resonance imaging. IEEE Trans Med Imaging 23:137-152.

Buckley MJ, Gaffan D (2006) Perirhinal cortical contributions to object perception. Trends Cogn Sci 10:100-107.

Buckley MJ, Booth MC, Rolls ET, Gaffan D (2001) Selective perceptual impairments after perirhinal cortex ablation. J Neurosci 21:9824-9836.

Buffalo EA, Reber PJ, Squire LR (1998) The human perirhinal cortex and recognition memory. Hippocampus 8:330-339.

Buffalo EA, Ramus SJ, Clark RE, Teng E, Squire LR, Zola SM (1999) Dissociation between the effects of the damage of perirhinal cortex and area TE. Learn Mem 6:572-599.

Bussey TJ, Saksida LM, Murray EA (2002) Perirhinal cortex resolves feature ambiguity in complex visual discriminations. Eur J Neurosci 15:365-374.

Bussey TJ, Saksida LM, Murray EA (2003) Impairments in visual discrimination after perirhinal cortex lesions: testing "declarative" vs. "perceptualmnemonic" views of perirhinal cortex function. Eur J Neurosci 17:649-660.

Crawford JR, Howell DC (1998) Comparing an individual's test score against norms derived from small samples. Clin Neuropsychol 12:482-486.

Devlin JT, Price CJ (2007) Perirhinal contributions to human visual perception. Curr Biol 17:1484-1488.

Epstein RA (2008) Parahippocampal and retrosplenial contributions to human spatial navigation. Trends Cogn Sci 12:388-396.

Epstein R, Kanwisher N (1998) A cortical representation of the local visual environment. Nature 392:598-601.

Hartley T, Bird CM, Chan D, Cipolotti L, Husain M, Vargha-Khadem F, Burgess N (2007) The hippocampus is required for short-term topographical memory in humans. Hippocampus 17:34-48.

Insausti R, Juottonen K, Soininen H, Insausti AM, Partanen K, Vainio P, Laakso MP, Pitkänen A (1998) MR volumetric analysis of the human entorhinal, perirhinal, and temporopolar cortices. AJNR Am J Neuroradiol 19:659-671.

Jenkinson M, Smith S (2001) A global optimisation method for robust affine registration of brain images. Med Image Anal 5:143-156.

Jenkinson M, Bannister P, Brady M, Smith S (2002) Improved optimization for the robust and accurate linear registration and motion correction of brain images. Neuroimage 17:825-841.

Kanwisher N, McDermott J, Chun MM (1997) The fusiform face area: a module in human extrastriate cortex specialized for face perception. J Neurosci 17:4302-4311.

Lee ACH, Bussey TJ, Murray EA, Saksida LM, Epstein RA, Kapur N, Hodges JR, Graham KS (2005a) Perceptual deficits in amnesia: challenging the medial temporal lobe "mnemonic" view. Neuropsychologia 43:1-11.

Lee ACH, Buckley MJ, Pegman SJ, Spiers H, Scahill VL, Gaffan D, Bussey TJ, Davies RR, Kapur N, Hodges JR, Graham KS (2005b) Specialisation in the medial temporal lobe for processing of objects and scenes. Hippocampus 15:782-797.

Lee ACH, Buckley MJ, Gaffan D, Emery T, Hodges JR, Graham KS (2006) Differentiating the roles of the hippocampus and perirhinal cortex in processes beyond long-term declarative memory: a double dissociation in dementia. J Neurosci 26:5198-5203.
Lee ACH, Levi N, Davies RR, Hodges JR, Graham KS (2007) Differing profiles of face and scene discrimination deficits in semantic dementia and Alzheimer's disease. Neuropsychologia 45:2135-2146.

Lee ACH, Scahill VL, Graham KS (2008) Activating the medial temporal lobe during oddity judgment for faces and scenes. Cereb Cortex 18:683-696.

Levy DA, Shrager Y, Squire LR (2005) Intact visual discrimination of complex and feature-ambiguous stimuli in the absence of perirhinal cortex. Learn Mem 12:61-66.

Malach R, Reppas JB, Benson RR, Kwong KK, Jiang H, Kennedy WA, Ledden PJ, Brady TJ, Rosen BR, Tootell RB (1995) Object-related activity revealed by functional magnetic resonance imaging in human occipital cortex. Proc Natl Acad Sci U S A 92:8135-8139.

Murray EA, Bussey TJ (1999) Perceptual-mnemonic functions of the perirhinal cortex. Trends Cogn Sci 3:142-151.

Murray EA, Bussey TJ, Saksida LM (2007) Visual perception and memory: a new view of medial temporal lobe function in primates and rodents. Annu Rev Neurosci 30:99-122.

Olson IR, Moore KS, Stark M, Chatterjee A (2006) Visual working memory is impaired when the medial temporal lobe is damaged. J Cogn Neurosci 18:1087-1097.

O'Neil EB, Cate AD, Köhler S (2009) Perirhinal cortex contributes to accuracy in recognition memory and perceptual discriminations. J Neurosci 29:8329-8334.

Ranganath C, D’Esposito M (2001) Medial temporal lobe activity associated with active maintenance of novel information. Neuron 31:865-873.

Rorden C, Brett M (2000) Stereotaxic display of brain lesions. Behav Neurol 12:191-200.

Schacter DL, Cooper LA, Delaney SM (1990) Implicit memory for unfamiliar objects depends on access to structural descriptions. J Exp Psychol Gen 119:5-24.

Shrager Y, Gold JJ, Hopkins RO, Squire LR (2006) Intact visual perception in memory-impaired patients with medial temporal lobe lesions. J Neurosci 26:2235-2240.

Smith SM, Jenkinson M, Woolrich MW, Beckmann CF, Behrens TE, Johansen-Berg H, Bannister PR, De Luca M, Drobnjak I, Flitney DE, Niazy RK, Saunders J, Vickers J, Zhang Y, De Stefano N, Brady JM, Matthews PM (2004) Advances in functional and structural MR image analysis and implementation as FSL. Neuroimage 23 [Suppl 1]:S208-S219.

Squire LR, Stark CE, Clark RE (2004) The medial temporal lobe. Annu Rev Neurosci 27:279-306.

Stark CE, Squire LR (2000) Intact visual perceptual discrimination in humans in the absence of perirhinal cortex. Learn Mem 7:273-278.

Tsivilis D, Vann SD, Denby C, Roberts N, Mayes AR, Montaldi D, Aggleton JP (2008) A disproportionate role for the fornix and mammillary bodies in recall versus recognition memory. Nat Neurosci 11:834-842.

Watson C, Jack CR Jr, Cendes F (1997) Volumetric magnetic resonance imaging. Clinical applications and contributions to the understanding of temporal lobe epilepsy. Arch Neurol 54:1521-1531.

Williams P, Tarr MJ (1997) Structural processing and implicit memory for possible and impossible figures. J Exp Psychol Learn Mem Cogn 23:1344-1361.

Woolrich MW, Ripley BD, Brady M, Smith SM (2001) Temporal autocorrelation in univariate linear modeling of FMRI data. Neuroimage 14: 1370-1386. 